Meta

Journal des traducteurs

Translators' Journal

\title{
Quality Factors in Documentary Translation
}

\section{María Pinto}

Volume 46, numéro 2, juin 2001

Évaluation : paramètres, méthodes, aspects pédagogiques /

Evaluation: Parameters, Methods, Pedagogical Aspects

URI : https://id.erudit.org/iderudit/003840ar

DOI : https://doi.org/10.7202/003840ar

Aller au sommaire du numéro

Éditeur(s)

Les Presses de l'Université de Montréal

ISSN

0026-0452 (imprimé)

1492-1421 (numérique)

Découvrir la revue

Citer cet article

Pinto, M. (2001). Quality Factors in Documentary Translation. Meta, 46(2), 288-300. https://doi.org/10.7202/003840ar

\section{Résumé de l'article}

Parce que nous sommes consciente des difficultés pour intégrer les modèles de traduction et systèmes de qualité, nous présentons un échantillon des développements récents, tout particulièrement en ce qui concerne la traduction pragmatique et les aspects méthodologiques en assurance de qualité. 


\title{
Quality Factors in Documentary Translation
}

\author{
MARía PINTO \\ University of Granada, Granada, Spain
}

\section{RÉSUMÉ}

Parce que nous sommes consciente des difficultés pour intégrer les modèles de traduction et systèmes de qualité, nous présentons un échantillon des développements récents, tout particulièrement en ce qui concerne la traduction pragmatique et les aspects méthodologiques en assurance de qualité.

\begin{abstract}
Well aware of the difficulties involved in integrating translating models and quality systems, we offer an overview of relevant developments in the field. Particular emphasis is placed on the pragmatic connotations of translation and on the methodological aspects of the Quality Paradigm, an approach to documentary translation that focuses activity on the target user.
\end{abstract}

\section{MOTS-CLÉS/KEYWORDS}

quality systems, translating models, pragmatic, Quality Paradigm, documentary translation

\section{Translation from a Functionalistic Standpoint}

The conception of Translation as a scientific activity is a reality emerging at the crossroads of paradigms and models. The common ground that was once occupied by the original linguistic model is now addressed by approaches of a pragmatic nature which helps our understanding of this phenomenon as it gains in complexity with the introduction of textual and situational aspects. The important contribution of these models is that they have changed the focus from translation as text reproduction to text production (Schaffner 1998: 1). The product of the translation process can no longer be deduced univocally from the source text, depending on specific expectations and needs of the target audience. The function of a text is its use or application in a given context or situation. Functionalism affirms that there is no absolutely correct translation outside that context, and makes the traditional notion of linguistic equivalence obsolete: it is more appropriate to speak of functional equivalence. For these reasons, we must learn to think in terms of translating strategies, and decision-making, instead of norms and principles. The links or relations between the textual unit, the immediate context, the superior context, the function of the source text and the function of the target text in its specific cultural situation must all be taken into account, at all times. Both the translator and the target reader will benefit from the advantages of this perspective, as it gives them both much more influence in the translating activity.

From a functionalistic perspective, the objectives of translation as defined in the assignment at hand will determine the method to be used in order to achieve the maximum level of communicative acceptability, a concept that is placed in opposi- 
tion to the classic concept of linguistic equivalence. The limitation of such possible objectives is the exclusive responsibility of the translator.

The development of functional translation is a complex process conditioned by a series of variables: most important, the objectives, objects, contexts, subjects, and strategies implied.

\section{Objectives}

The function of the target text depends directly and fundamentally on the expectations and the needs of its readers. It is they who determine the objectives of the translation. For this reason, we must elucidate the communicative situation by defining —at least—-the following points:

1) function of text

2) user profile

3) time-space limitations of the target context, and

4) type of transmitting document.

\section{Objects}

The text is an obligatory point of departure in translating processes, by virtue of its capacity of response to the informative-communicative needs of the human species. Interpersonal communication takes place preferentially by means of the complex textual units, and the concepts associated with them depend on to a great extent on the point of view adopted for their study.

\section{Contexts}

There exists no absolutely correct translation out of context. Any analysis of content-and translating processes are just that — should be carried out with regards to the situational contexts (origin and target) and should be justified itself in terms of these, as content is not an a priori function of the properties of documents. Sense (what is understood) is a many-faceted phenomenon, being communicative as well as pragmatic and psychological, the product of a text plus a situation. The transformation of meaning (denotation) into sense requires putting the text into a context (situation). One important problem facing the translator as the intermediary between two languages and two cultures is knowing just what part of these contexts should be reconstructed, avoiding the superfluous, and/or elaborating the implicit information that is necessary for a correct interpretation of the Target Text (TT).

\section{Strategies}

Translation as the Processing of Information. The processes involved in translating have, as their objective, the replacement of a text in the source language with a message in the target language that is semantically and pragmatically equivalent. Here is where the full nature of the translator comes into play. Not only must he gather, filter and analyze the documentation for the topic of the translation, but he must also play a technical role, showing good command of the original text, the process of translation per se, and the elaboration of the target text. In this sense, we consider translation as 
an operation of content analysis, with special predominance of textual and pragmatic analysis. This idea transcends the conventional notions of content as the object of study; indeed, it is closely linked with more recent conceptions of symbolic phenomena, in the sense of a distinct awareness of human communication, and of new means of communication and the role they play in the transmission of information (Krippendorff 1990: 10). The changes produced in the social scenario call for a structural definition of content to take into account all the channels and the restrictions of information flow, and the functions and effects of communicative processes within society.

The problem arises with the extreme difficulty derived from the many mysteries that surround, on the one hand, the complex textual mechanism and, on the other, the concept of content as the starting point on a specifically documentary journey (Pinto 1994: 113). This is a many-sided phenomenon that depends, among other things, on the attitudes of the speakers and listeners toward reality. For this reason, in addition to accounting for the substantial, descriptive, and intrinsic character of the texts, we must take into account their interactive (pragmatic, expressive) character, which is very closely related with the communicative intentions of the sender. Actually, the enunciative content is not an "intrinsic quality" of the text (Hjorland 1992: 181). This is rather a quality that is determined by the sending context and-above all-the receiving context.

Accordingly, the translator must be a dynamic entity, and as such dispose of his/ her own strategies to carry out the assigned task: the reading/comprehension of the original document, and the interpretation and production of the translated text. The readability of a text implies a balanced application of cognitive and meta-cognitive strategies, with the fundamental objective of comprehension, a process that depends essentially on the inferences made by the reader in interaction with the text. These inferences allow the reader to establish connections between the diverse textual elements, and integrate the explicit and implicit information with previous knowledge.

Once the information is comprehended, the text should be interpreted; that is, it will be assigned an adequate sense. The success of this interpretative act depends on the symmetry or asymmetry between the processes of production and interpretation, which involve two individuals with different competencies (communicating and interpreting).

\section{The Quality Paradigm}

The early notions of the concept of quality were centered on the fulfilment of certain specifications; this conception gave way to the philosophy of suiting individual needs, to end up with user satisfaction as the basic principle.

The systems of Quality Management (QM) aspire to ensure the complete satisfaction of users, personnel, business managers, and society in general. Quality is understood as quality at all levels: from the conception of a product to quality in its production, along with procedures for quality control, and quality in the service that accompanies the product. These QM systems pursue quality as it is defined in terms of customers' perceptions (Rowley 1996: 17). The involvement of personnel is enhanced by the promotion of an overriding aim of continual improvement, and the goal of error-free translating processes. 
Based on this global approach to quality, the European Model EFQM (European Foundation of Quality Management) is useful for any organization that hopes to introduce, develop and control a "culture of Quality." Essentially, the message is that Excellence, or the state of generalized maximum satisfaction, depends on nine integral factors. Five of these constitute the tools for Quality, and the other four are the consequences of the adoption of a Quality policy. Excellence in Translating Services is the result of the necessary integration of these fundamental ingredients in the planning stage of any such undertaking. Overall Performance, in accordance with the criteria of planning, self-evaluation or comparison described here, would be expressed numerically, by a total of the points assigned to each criterion. This conceptual diagram is highly significant: it provides for a very precise definition of the variables involved in the phenomenon of Quality, while also assigning a percentage value to each of these interrelated activities and concepts. Thus, they acquire relative weight in the service as a whole. The holistic nature of Total Quality means that all of them are necessary conditions.

\subsection{Client Satisfaction}

In the face of increasing competition and the globalization of the different sectors of the economy, the European Union is working to develop the "European Index of Client Satisfaction." This Index would constitute a fundamental tool for decisionmaking and strategy planning among member countries. Excellent results have been seen under similar projects carried out in Sweden (since 1989), Germany (1992), and the US (1994). Indeed, the countries of the European Union (including Spain) are already taking part in the Pilot Project—-the EURIX Program-to introduce the Client Satisfaction Index.

With client satisfaction regarded as the means of measuring quality in Translating services, we need to determine Quantitative characteristics: delays, scheduling, number of visits needed to solve a problem, deadlines; Qualitative characteristics: favorable atmosphere, courtesy, friendliness, confidence, guarantees of service; Service characteristics: the duration of the process from its request until its finalization, the capacity of response in the face of unexpected events, persons who intervene in the service, complementary elements, system of lodging complaints, provider-professional-client communication, competence of the persons involved, reliability of service and mutual satisfaction achieved.

But the vast amount of work done thus far to measure user satisfaction was disappointing, in part because the global means of measuring satisfaction rarely point to the road to action for attaining improvement. Moreover, it is very difficult to quantify in reproducible terms the "soft" aspects of satisfaction, or the relation existing between satisfaction and demand (Brophy 1995: 77). In any case, we must admit that only the redefinition - in a more inclusive sense-of the client/user term would allow these objectives of QM to be reached.

\subsection{Staff Satisfaction}

Although Quality Management has traditionally been centered on the client/user, the proposed European Model opens up this concept to include all those persons with an 
interest in the organization and in its activities. Again, the individuals who work for a Translating Service (staff) are also users, and as such form an integral part of the Quality production line. Au moment où l'emphase est mise sur les nouvelles technologies de l'information, il peut être tentant de n'évaluer les services d'information qu'en termes de performance des moyens techniques offerts aus usagers. Pourtant, la qualité dépende également de la façon dont les employés accomplissent leurs tâches. (Bouthillier 1993: 31)

\subsection{Leadership}

The concept of leadership refers us to the individual or group responsible for directing the Translating Service under the set of principles of Quality Management. The leader (person or team) should inspire, support and promote this culture, and will assume and/or elaborate the Regulations of the Translation Service as the normative framework and element of control for setting objectives and goals. Leadership will facilitate the integration of all the key Service criteria in the continual search for Quality improvement, and will be in charge of providing appropriate resources and assistance. Leaders will be committed to the clients, providers and other external organizations, listening to their input in order to promote innovation (Austiin, Peters 1986: 63).

\subsection{Staff Management}

The Translating Service should fully develop the potential of its staff for the sake of constant improvement. In this sense, it is important that the human resources be well planned and provided for, that appropriate recognition and authority are extended to them, and that their capacities are continually developed through training. Personnel must agree on overall objectives and periodically review their own performance. Their full participation in the process of continual improvement means that they maintain effective dialogue with the organization.

Adequate training of staff members implies much more than "know-how" or competence, and embraces excellence in personal achievement: attitude, motivation, conduct, perceptions and human relations are primary considerations. When the concepts of autonomy, responsibility and commitment are upheld among staff members, they will find it easier to develop their potential, from their different work positions, for the sake of attaining a higher degree of individual and collective excellence.

A clearly defined Personnel Policy is needed to establish the adequate degree of preparation and the appropriate assignment of functions and levels of responsibility and to develop integral training programs in accordance with the organization's global policy and standards. Staff changes, salary raises, and high-level training programs would be planned for. And because the Quality of Service is a matter of output as well, personnel performance would be analyzed regularly by assigning a set of activities whose realization and influence on performance could be observed and objectively measured.

Human resources should be the center of our concern as managers of translating services, with a constant awareness of the fact that human satisfaction at any given 
point in the process as a whole can only be guaranteed when a harmonic interaction exists between all the different factors involved.

If we are serious about providing quality services, we need to learn from the expectations of quality held by users, in accordance with a democratic perspective oriented to the achievement of common objectives in the interest of the community in general. The idea is to negotiate-ahead of time-the services to be delivered, in order to make the best possible use of resources. In this way, translating services are offered "intentionally," in coherence with the previously determined group of users.

But the unquestionable reality is that processes, products and translation services have never been defined in a unanimous and satisfactory way, and this collides with the Quality Paradigm, which demands regulation, precision, quantification and assessment as indispensable conditions for its effective implantation in any setting. We will take the opportunity to mention the premises for a definition of the policy of such centers. The establishment of a Quality System in a Translation Service would oblige us to:

- Delimit the source and target cultural and situational spaces.

- Define objects (text types).

- Define subjects: Active (translator) and Passive (user).

Listening to "the voice of the user" is the very first step to getting to know his wishes, and the final means of measuring satisfaction. The user "wishes" constitute a broad concept that lends itself to an arrangement in different categories, within a hierarchical scheme. Learning about these "wishes" is a prerequisite for their transference to the technical language of translation:

- Establishing textual functions

- Establishing (formal) Prerequisites and (functional) Performances.

\section{Documentation at the Service of the Translator}

The ever-increasing documentary outpour, conveniently channeled by the implantation of analysis systems at both a general and a domestic level, afford the translator access to a greater amount of information in a shorter time period. Consequently, the translator's levels of efficiency and effectiveness are appreciably higher.

The Translator is the key to the process of translation, as author of a new document that is representative of the source text, and-most importantly-destined to fulfill a specific function in the receptor context. The interest and knowledge of the translator, who should know for whom and for what purpose he is translating, will determine the construction of the aforementioned context. But the lack of a proper assessment of the work carried out by these professionals spurs us to renovate the prestige of their professional activity, and look up to it as an intellectual effort of the first degree (Ortega y Gasset 1958: 451). Functionalism makes a strong contribution in this sense, with its focus on the translator as the true protagonist of the processes; this orientation grants the translator more freedom while assigning him, likewise, a greater degree of responsibility.

The original document takes on meaning through the organized structures, or "frames," of the translator as information analyst. These frames of reference take the form of complex semantic networks in the translator's memory that embrace all his 
knowledge, beliefs and feelings with respect to himself, or other persons or events, in episodic, semantic or affective terms. But the framework will hardly be sufficient for the textual processing of the text, so that the translator, to some extent, will have to resort to organization and documentary techniques as indispensable assistants in the task. His linguistic, cognitive, scientific and functional competence must be complemented with documentary skills.

At this point in our analysis, we must address an apparent contradiction inherent in the translator's role despite being an especially demanding user of documentary services (Williams 1996: 276). He does not know how to gather documentary material properly. It goes without saying that documentary assistance is fundamental, in view of the multiplicity of tasks to which these professionals are subjected. The documentary competence of the translator is deployed in three facets of his task: as user, as processor and as producer of documents.

\subsection{The Translator as User of Documents}

Studying documents whose topic is similar to that of the text to be translated in both of the languages involved, prepares the translator for delving into the assigned text (Maillot 1997: 231). This phase of documentary search and retrieval will therefore condition, to an appreciable extent, the processes and the product of the translation. It is a case of selecting the ideal information by discerning information sources that may be unnecessary or outdated. The choice of resources will depend on economical considerations, the nature of the material, the origin of the publications and the thematic interest of the users. During this stage of information gathering, when the source text needs to be flanked by other documents that will provide a context, the figure of the documentalist as a documentary advisor takes on special significance, regardless of differences between the two models of search-retrieval: traditional and computer-based.

The range of documentary products at the service of the translator includes everything from original and secondary documents (catalogues, indexes, summaries and abstracts, databases and more) to the tertiary documents such as encyclopedias, dictionaries, and specialized vocabulary lists. All these tools-which are doubled in number in the source and target contexts and cultures-are indispensable for the translator. This support should be methodical in order to be efficient. The documentary aid that we propose should be used in both the source and target languages and cultures, and should be developed not only in the semasiological stage of text comprehension but also in the onomasiological stage of assigning the most appropriate terms to these elements of comprehension

References: Parallel documents are a resource that are, quite frankly, underestimated and under-utilized. These bibliographic references, however, give more detailed and more appropriate information than dictionaries, and should never be overlooked.

Terminologies: They are also key, above all in the case of specialized documents that are only accessible through the comprehension and use of technical terms. The conditions surrounding the translating activity, particularly the time restrictions, do not permit the translator to acquire a complete body of knowledge of all the notions evoked in a specialized text. The textual trek in and out of bilingual and monolingual 
dictionaries, encyclopedia, and thesauruses that is imposed on the translator is very well suited to hypertextual vehicles. The computerized hypertext or hypermedia innovations can improve the performance of translators not only in the qualitative sense (translation quality) but also in quantitative aspects (shorter time periods needed to complete assignments).

Encyclopedia: Assuming that the degree of comprehension is the key to all translating, and in particular to that of specialized texts, we must underline the high value of the encyclopedia among translators. There are two reasons for this: an encyclopedia presents an enormous quantity of knowledge in a very well ordered and structured manner, and, it lends itself to free consultation according to the tastes of the reader, who may sail the seas of data presented as it suits his needs. Moreover, because a degree of comprehension is reached not only insofar as concepts are concerned, but also with respect to the relations among these concepts, we must continue to consider encyclopedia as high priority documentary tools. They explain processes, phenomena, and mechanisms and, in a way, place them on stage, describing their natural and real context. This facilitates the establishment of superior relationships, something that the monolingual thematic dictionaries cannot do with their isolated entries. The encyclopedia contributes more than any other documentary aid to obtaining what some experts call the autonomous system of knowledge. This integration of concepts and their relationships in a structured whole ensure a state of comprehension that will suffice to proceed with the translating process per se in a reliable manner.

Dictionaries: Despite certain great advantages that make them the first and most immediate aid used, all printed dictionaries share the disadvantage of size restrictions and outdated contents. Moreover, most are ordered alphabetically, making them weak in representing multi-word units (Balkan 1992: 409).

\subsection{The Translator as a Processor of Information}

A strategic model of the translating operation should combine the processes of construction of the communicative message from the source text: ascendant information, or "bottom-up" proceedings; the integration of this information with the previous knowledge stored in the memory of the translator; descendant knowledge, or "top-down" proceedings; and the interaction of the two aforementioned processes with the objectives and contexts of the translation.

Strategies, plans of action at the service of translation processes, lead to a series of activities or mental operations (selection, organization, processing and production) that enhance the comprehension, interpretation and production of the documentary content. We must remember that the active role of the translator in the processing of information is not based on a utopic series of rules but depends on the reality of the strategies, operations or mental activities that facilitate and develop the translating processes. These must allow the translator to process, organize, retain and retrieve the initial information, and then help him to plan, regulate and evaluate those same processes with respect to previously outlined objectives. A strategy can be seen as a series of competencies, a set of steps or processes that favor the acquisition, storage and/or utilization of information. Strategies are heuristic and flexible in nature, and their adoption implies a decision influenced not only by amendments in the translator's objectives, but also by the structural characteristics of the assignment at 
hand. They may also depend on what is sometimes called "procedural knowledge"; that is, they refer to action-oriented skills, which depend largely on the individual qualities and interests of the translator.

Human communication revolves, basically, around what exists beyond the boundaries of discourse. The bulk of the information we transmit, or which is transmitted to us, is implicit, not explicit. Therefore, as Schank explains, "inference is the nucleus of the process of comprehension, [...] constituting the center of the realm of human communication, serving to closely unite entries of information into an interrelated whole. Often, the inferences themselves are the main point of the message" (Schank 1979: 187). Actually, the processes that connect textual contents and the translator's previous knowledge are inferences of different types. Even during the first stage of reading, it is assumed that the memory of the translator activates his schemes for deriving conclusions that extend beyond the text at hand (Ballstaedt, Mandl 1984: 331).

In consonance with this obligation of the professional translator to serve as and be served by a permanent inferential strategy, a broad base of general knowledge is crucial, in addition to a specific knowledge of the subject area of the documents to be translated. This training will help him to fill in those "gaps" in the subject matter that are vital for a correct interpretation of discourse. Van Dijk \& Kintsch (1983: 51) proposes that inferences are cohesive mechanisms pertaining to a separate level of analysis: not textual, but situational. They also help eliminate ambiguity, as propositions that are closely related semantically can be clearly distinguished by inferential interpretations. Likewise, inferences may establish the linguistic context when material circumstances, the situational environment or the time/space coordinates of discourse are absent.

\subsection{The Translator as the Producer of Documents}

The final result of any translating process is a written text that reflects a) the tactic, rhetorical and functional competence of the translator in the process of re-elaborating documentary information, b) the capacity of sequential and logical text execution on the part of the translator, and, to some degree, c) the effectiveness of the final expression of content. In general, the activity of writing involves a series of flexible and interactive components. These serve to gather up and integrate previous knowledge, linguistic and organizational restrictions, new knowledge on the subject and modes of discourse compatible with the communicative context.

The profile of target text writing (Endres-Niggemeyer 1989: 104) that we focus our attention on here is derived from the cognitive model (Flower, Hayes 1981: 366).The main component is "the task environment," in reference to the operative context in which the translating process is carried out. There are various influencing factors: the activation of certain processes will be different depending on the type of original document (report, article, book); the extraction of contents from the original document will be conditioned by the type of translation (overt/covert, text function) that is intended, or the readership to which it is directed. In addition, we must consider the working conditions: the available tools or other means of making the target text materialize, time allowances, and the amount of text produced up to the moment. 


\section{The User as the Judge of Translation Quality}

From the Quality Management standpoint, all aspects of the enterprise must be looked after: the quality of the enterprise, of the translator, of the processes employed, of the translation itself, and even of the reader. These ingredients should all fulfill certain essential characteristics of Quality. According to ISO norm 9000, these are the elements that can affect the quality of the translation product. The company, organization, or translating service should establish its own models and methods of planning and management, in view of its levels of general and technological resources.

From a perspective of combining the Functionalistic trend in translation with the Quality paradigm, the reader (the user, the receiver of the target text) is the main agent in swaying the decisions of the enterprise and the translator. There is no denying that the quality of the translation is a perception that depends directly on the degree of satisfaction reached by its readers. A previous study of the user profile of a specific translation, of user expectations and needs, is the groundwork on which the specific objectives of a translation must be founded. In turn, it serves as the foundation for the translating strategy as a whole.

In accordance with the general principle of communicative acceptability that presides over the functionalist current in translation, we will suggest a series of attributes for the derived product of the translation processes (the target text) that will give us a point of departure in arriving at parameters for qualitative assessment. The effectiveness of the multi-criteria analysis in evaluating a product is well established in other sectors.

\subsection{Cognitive Representativity in Translation}

A quality translation should offer a certain level of cognitive correlation with the source text, although this aspect depends on the type of translation, as predetermined in the light of the distance between the source and target contexts. The lesser the intercultural distance, the greater the correlation. Maximum correlation occurs when the two cultures coincide, as in scientific documents. Depending on the intercultural separation, some optimal levels of correlation can be established, to be evaluated by means the indicators described below.

Accuracy or precision. It would give us an approximate idea of the success of the translator in dealing with the text overall, allowing us to check the adaptation to the source text and the inclusion, or omission, of extra-textual information. Needless to say, precision will be affected by the characteristics of the text itself and by the degree of comprehension reached by the translator. Relevant aspects of accuracy include: the correct choice of technical terms, absence of accidental omissions, and care taken in the interpretation of ambiguities, of repetitious passages, of idiosyncratic expressions of the author, and of errors. The latter can be typified in the following manner: inversion of meaning, omission, addition, deviation, and modification. Their effects on the text, as the gauge of maximum user satisfaction, can vary in magnitude. Pragmatic errors occupy the most significant position in the hierarchy, as they violate the pragmatic instructions of the translating assignment; and they are followed in importance by cultural or linguistic errors. 
One important measurement of quality should be the clarity and readability of the final product. The readability of a text is determined by a combination of factors that make it comprehensible, and can be assessed using tests based on specific indicators. Different computer programs now on the market assess readability by giving a final score to each text. In any case, a high percentage of passive constructions, excessive prepositions, too many sentences per paragraph, too many words per sentence, and too many syllables per word all make reading more difficult.

Bearing in mind that any translation is, or should be, an authentic text, it must fulfil two fundamental prerequisites of textual units in general: cohesion and coherence. Cohesion refers to a superficial linking, a lexical-grammatical union, and requires all the propositions to be syntactically coordinated. An elementary mode of measuring the degree of cohesion of translations would be to establish the relationship between the number of proposals connected superficially in a certain target text with the total number of propositions of the corresponding source text. Nonetheless, coherence resides in the deep structure of the text, and for which each one of the textual sequences will be comprehensible as pertaining to the same subject. One simple way of estimating coherence would be to compare the number of propositions of the text that are considered pertinent to the subject with the total number of propositions overall.

\subsection{Functional Adequacy of the Translation}

Above all, the Quality of a translation should be measured in terms of the level of adherence to the predetermined objectives.

According to functionalism, the definition of the type of target text becomes the objective of the translation, and a clear definition of objectives-that is, the functionality of the target text-would allow evaluators to confirm the respective degree of fulfilment.

Consistency is essentially a measure of the similarity of the reactions of different individuals when processing the same information. We may define the consistency of a target text as the ratio between the number of identical propositions used to translate one same source document by two different translating operations, and the number of different propositions used in the whole of these two operations. We shall distinguish between personal consistency and team consistency. Among the factors involved in this indicator, we find text quality, the existence of translating strategies, the correct comprehension of the text, and the (consensual) awareness of the needs of the users on the part of the translators.

\section{Conclusions}

The translator is the nucleus of translating operations. Given his importance in the context of a functional approach to such operations, the documentary support of his activity should be given special care: firstly, by supplying more and better documentary tools (original documents, references, glossaries, encyclopedia, dictionaries), and secondly, by improving his degree of documentary formation, not only as a processor of information, but also and above all, as a user of a practically unlimited documentary orbit. 
The introduction of new technologies (computers and telecommunications, an all-important area where these two converge) has altered the scenario of the professional translator (Theologitis 1998: 342). The translator can use these innovations to improve his professional productivity and ergonomics. The presence of the computerized documentation in the translating setting is immensely beneficial in a material sense: personal computers, automated translating techniques or aids, and access to multilingual documents are the most familiar contributions.

Having defined the means, process and results of the Quality System, we may proceed to its implantation in the Translating Service, beginning with a sincere selfevaluation and a rigorous process of planning that would keep the following ideas alive.

- The textual function directly related with the text typologies is the key ingredient of the translating objectives.

- These objectives are significant in determining the translating processes.

- The key to the process is the translator.

- The philosophy of Quality, in accordance with the functionalist trend, requires that a user-oriented approach be applied to all the activities involved in the translating service.

In the face of the difficulties involved in the establishment of criteria for measuring the quality of the processes, products and services of translation, the functional tendency facilitates the road to reliable and stable indicators. L'analyse multi-critères est fondée sur le principe qu'il n'existe aucune solution unique, représentant un excellent moyen d'évaluer les différentes solutions à un problème en fonction d'un ensemble de critères pondérés, car il obligue l'analyste à se poser des questions sur les critères euxmêmes et à leur accorder une valeur relative. (Larose 1994: 367).

We insist, finally, that Quality is a matter of orientation, of leadership, of employee participation and proper training. Under any circumstances, quality improvement is a never-ending process that must be undertaken one step at a time, and is not likely to offer immediate results.

\section{REFERENCES}

Austin, N., Peters, T. (1986): Pasión por la excelencia. Méjico, Lasser Press, 1986.

BALKAN, L. (1992): Translation Tools. META, 37, 3, pp.408-420.

Ballstaedt, S., H. Mandl (1984): Elaborations: assessment and analysis. In: Mande, H., N. Stein, T. Trabasso (eds.), Learning and comprehension of text. London, Lawrence Erlbaum Associates.

Bouthillier, F. (1993): Services d'information et evaluation du rendement du personnel. Argus, 22, 2, pp. 31-34.

Brophy, P. (1995): Quality Management in Libraries. Proceedings of the 1st Northumbria International Conference. Performance measurement in Libraries and Information Services. Newcastle: University of Northumbria, pp. 77-81.

Endres-Niggemeyer, B. (1989): Content analysis: a special case of text comprehension. In Proceedings: Information, Knowledge, Evolution. 44 FID Congress. Amsterdam, Elsevier.

Flower, L., J.R. Hayes (1981): A cognitive process theory of writing. College Composition and Communication, 32, 365-387.

Hjorland, B. (1992): The concept of "subject" in information science. Journal of Documentation, 48, 2, pp. 172-200. 
International Standard Organization (1977): Norm ISO 2384. Documentation. Presentation of Translation. Geneve, ISO.

Krippendorff, K. (1990): Metodología de análisis de contenido. Teoría y practica. Barcelona: Paidos.

LARose, R. (1994): Qualité et efficacité en traduction: réponse à F.W. Sixel. META, 39, 2, pp. 362373.

Maillot, J. P. (1997): La traducción científica y técnica. Madrid, Gredos.

Nord, C. ( 1996): El error en la traducción: categorías y evaluación. En: Hurtado, A. (ed.) La enseñanza de la traducción. Castellón, Universidad Jaume I.

Ortega Y Gasset, J. (1958): Miseria y esplendor de la traducción. Obras Completas. Madrid, Revista de Occidente, $4^{\text {a }}$ edición, tomo V.

Pinto, M. (1994): Interdisciplinary approaches to the concept and practice of written text documentary content analysis (WTDCA). Journal of Documentation, 50, 2, pp. 111-133. Award FID/MIP (Modern Information Professional) as the best article of the year.

Rowley, J. ( 1996): Implementing TQM for library services: the issues. ASLIB Proceedings, 48, 1, pp. 17-21.

Schaffner, C. (1998): From "Good" to "Functionally appropriate": In: Schaffner, C. (ed), Translation and Quality. Clevedon, UK, Multilingual Matters, 1998.

Schank, R.C. (1979): El papel de la memoria en el procesamiento del lenguaje, In COFER, Ch, (dir.) Estructura de la memoria humana. Barcelona, Omega.

Theologitis, D. (1998): The Impact of New Technology on the Translator. Terminologie et Traduction, 1, pp. 342-351.

Van DijK, T.A., W. Kintsch, (1983): Strategies of discourse comprehension. New York, Academic Press.

Williams, I.A.(1996): A translator's reference needs: dictionaries or parallel texts? Target, 8, 2, pp. 275-299. 